\title{
Simultaneous Occurrence of Urothelial Bladder Carcinoma and Primary Retroperitoneal Leiomyosarcoma
}

\begin{tabular}{|l|l|l|}
\hline U. & Ursula & Herrmann \\
\hline A. & Avelino & Hernandez \\
\hline G. & Georg & Hofmockel \\
\hline H.G.W. & Hubert G.W. & Frohmüller \\
\hline
\end{tabular}

Department of Urology, University of Würzburg, Medical School, Würzburg, Germany

\section{Key Words}

Retroperitoneal leiomyosarcoma

Urothelial bladder carcinoma

\section{Abstract}

A case of primary carcinoma of the urinary bladder associated with a primary retroperitoneal leiomyosarcoma is presented. Due to the lack of early symptoms, diagnosis of the retroperitoneal leiomyosarcoma was late and therefore the prognosis was poor. Twelve months after diagnosis the patient died not of the bladder tumor, but of the recurrent leiomyosarcoma.

Dr. med. Ursula Herrmann, Department of Urology, University of Würzburg, Medical School, Josef-Schneider-Strasse 2, D97080 Würzburg (Germany), Tel.+49 93120 11, Fax+49 9312013816

Introduction

In 1879, Billroth first observed multiple independent primary neoplasms in an individual patient [1]. Since then, multiple primary malignancies have been described and their criteria for diagnosis are well defined today: (a) each tumor must present a definite pattern of malignancy; (b) each tumor must be distinct, and (c) the probability that one was a metastatic lesion from the other must be excluded [1, 2].

In the literature the incidence of bladder tumors associated with malignant tumors outside the urinary epithelium is reported to be $15.9 \%$ [2]. Though coexistence of urothelial cancer and leiomyosarcoma of the bladder is well documented [3-6], to our knowledge, the simultaneous occurrence of urothelial bladder cancer and primary retroperitoneal leiomyosarcoma has not yet been reported. 


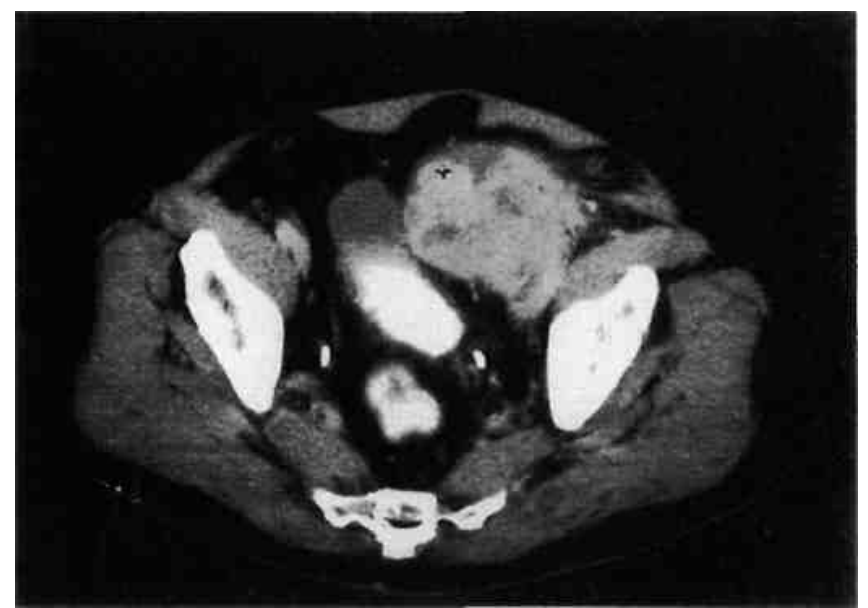

Fig. 1. Pelvic CT showing an inhomogeneous retroperitoneal mass on the left side, approximately $9 \mathrm{~cm}$ in diameter. The bladder is partly filled with contrast medium.

\section{Case Report}

W.A., a 68-year-old man, presented for urologic evaluation after a 2-week episode of obstructive uropathy and extending pain into the left groin. The physical examination revealed a painful palpable mass in the left lower abdomen, normal testicles and benign prostate hyperplasia diagnosed by digital rectal palpation. All variables in blood, including prostatic-specific antigen, were within the normal range. Microhematuria was present. Cystoscopy disclosed an exo-phytic tumor at the posterior wall of the urinary bladder. Both ultra-sonography and abdominal computed tomography (CT) confirmed the presence of an inhomogeneous, retroperitoneal tumor at the left-hand side, approximately $9 \mathrm{~cm}$ in diameter and without infiltration of any organ (fig. 1). Excretory urography showed a slight pyelocaliec-

\section{KAIUiË̈l}

E-Mail karger@karger.ch Fax+ 41613061234 http://www. karger. ch (C)1997S. KargerAG, Basel 0042-1138/97/0584-0252\$12.00/0

tasis of the left kidney and a trabeculated bladder but without any filling defects. Coloscopic examination was normal with no extrinsic masses noted. Thoracic CT was negative for metastases.

The lesion of the bladder was resected transurethrally. The histo-pathological diagnosis revealed a partly well, partly moderately differentiated noninfiltrating papillary carcinoma of the urinary bladder (stage pTa, malignancy grade I-II; WHO classification). One week later an explorative laparotomy was performed for resection of the tumor in the lower abdomen, showing the retroperitoneal origin of this mass. As no organs or vessels were involved, complete resection of this mass was possible. Histological examination revealed a poorly differentiated pleomorphic leiomyosarcoma (malignancy grade III) with unknown organ of origin. Four cycles of adjuvant chemotherapy containing ifosfamide and etoposide were administered postoperatively. Ten months later the patient presented with a recurrent retroperitoneal mass, which was inoperable. He died 2 months later.

\section{Discussion}

The genitourinary tract appears to have greater risk for multiple primary malignant neoplasms. Summarizing all data from several large surveys up to 1983, association of the urological organs with at least one second primary tumor was $13.5 \%$ [2]. Recently, in a series of 10,014 patients 
with bladder cancer, the incidence of subsequent primary cancer was reported to be $6.5 \%$ [8]. One-third of these second primary neoplasms occurs at the same time as the bladder tumor, while two-thirds are metachronous [7]. The latter are frequently localized in the prostate, skin and lip, colorectal area, bronchial system, breast, uterus and stomach $[2,7,8]$. However, to our knowledge the simultaneous occurrence of a primary urothelial bladder cancer and a primary retroperitoneal leiomyosarcoma has not yet been described.

Soft tissue sarcomas constitute less than $1 \%$ of all malignancies in adults, and in 15-20\% they are found in the retroperitoneum [9]. At this site, leiomyosarcoma is the second most common histological type of sarcoma [10]. Long-term prognosis of retroperitoneal leiomyosarcoma is poor, due to the lack of early symptoms resulting in late diagnosis. Regularly, the first clinical manifestation is a large abdominal mass. The histological grading is one of the most important prognostic factors and the frequency of local recurrence is high. Absence of multiple organ involvement provided, patients with low-grade sarcoma will benefit from complete resection as the only effective treatment available. In the literature, $10-50 \%$ of the retroperitoneal sarcomas are reported to be resectable $[11,12]$. Adjuvant radiation therapy and chemotherapy could not be proved to have significant influence on the survival rate [13]. In spite of having been treated by complete removal and adjuvant chemotherapy, our patient died of recurrent leiomyosarcoma 12 months after surgery. The clinical course was not determined by the bladder tumor, but by the retroperitoneal sarcoma. Despite the leiomyosarcoma's bulky nature and tendency toward local recurrence, in the case of concurrent malignancies proper therapy and prognosis depend on accurate diagnosis and follow-up of each tumor.

References

Moertel CG: Multiple primary neoplasms. Historical perspectives. Cancer 1977 (suppl 40): 1786-1792.

Matzkin H, Braf Z: Multiple primary malignant neoplasms in the genitourinary tract: Occurrence and etiology. JUrol 1989;142:1-12. Melicow MM: Tumors of the urinary bladder: A clinicopathological analysis of over 2,500 specimens and biopsies. J Urol 1955;74:498-521. Patterson TH, Dale GA: Carcinosarcoma of the bladder: Case report and review of the literature. J Urol 1976;115:753-755. Hejtmancik JH, Klatt WW: Coexisting carcinoma and sarcoma of the bladder. J Urol 1960; 84:320-321.

Mackles A, Immergut S, Grayzel M, Cottier ZR: Carcinoma and sarcoma of bladder: Report of unusual simultaneous occurrence of both tumors. J Urol 1948;59:1121-1126. Ward-McQuaid JN: Carcinoma of the bladder and multiple unrelated malignancy. Br J Urol 1963;35:169-172. Salminen E. Pukkala E. Teppo L, Pyrhonen S: Subsequent primary cancers following bladder cancer. Eur J Cancer 1994;30A:303-307. VanDoorn RC, Gallee MPW, Hart AAM, Gortzak E, Rutgers EJT, van Coevorden F, Keus RB, Zoetmulder FAN: Resectable retroperitoneal soft tissue sarcomas. Cancer 1994; 73:637-642.

Hartman DS, Hayes WS, Choyke PL, Tibbetts GP: Leiomyosarcoma of the retroperitoneum and inferior vena cava: Radiologic-pathologic correlation. Radiographics 1992; 12:1203-1220.

Rossi CR, Nitti D, Foletto M, Alessio S, Seno A, Segato G, Mancino G, De Marchi F, Ninfo V, Lise M: Management of primary sarcomas of the retroperitoneum. Eur J Surg Oncol 1993; 19:355-360.

Alvarenga JC, Ball AB, Fisher C, Fryatt I, Jones Thomas JM: Limitations of surgery in the treatment of retroperitoneal sarcoma. Br J Surg 1991;78:912-916. 
Jaques DP, Coit DG, Hajdu SI, Brennan MF: Management of primary and recurrent soft-tissue sarcoma of the retroperitoneum. Ann Surg 1990;212:51-59.

U. Urothelial Bladder Carcinoma and Primary Retroperitoneal Leiomyosarcoma 